Pacific Journal of Mathematic 


\title{
SIDON SETS ASSOCIATED WITH A CLOSED SUBSET OF A COMPACT ABELIAN GROUP
}

\author{
E. V. Dudley
}

\begin{abstract}
Déchamps-Gondim in [1] announced that a Sidon set $E$ contained in the dual of a connected compact abelian group $G$ is associated with each compact subset $K$ of $G$ having interior, in the sense that there exists a finite subset $F$ of $E$ and some constant such that this constant times the maximum absolute value of any $E \backslash F$-spectral trignometric polynomial on $K$ majorizes the sum of the absolute values of the Fourier transform. It is readily shown that if $G$ is not connected not all Sidon sets have this property. In [7], Ross described the class of all Sidon sets which are associated with all compact sets $K$ having interior. In this paper, the Sidon sets associated with a particular set $K$ are analysed and characterized.
\end{abstract}

\section{Introduction.}

1.1. Throughout this paper, the symbol $G$ is used to denote an arbitrary infinite, compact, abelian group, the symbol $X$ denotes its character group and $\lambda$, Haar measure on $G$. For $E$ a subset of $X$, we call an integrable function an $E$-spectral function if its Fourier transform vanishes off $E$. For any space $F(G)$ of integrable functions, the space of all $E$-spectral functions belonging to $F(G)$ is denoted by $F_{E}(G)$. We denote by Trig $(G)$, the space of all complex-valued trignometric polynomials on $G$ and by $A(G)$, the space of all functions with absolutely convergent Fourier series. The usual norm on $A(G)$ is denoted by \|\|$_{A}$. All other notation not explained in this paper appears in López and Ross [6].

Definition 1.2 (see López and Ross [6] p. 109). Let $K$ be a nonvoid compact subset of $G$ and $E$ a subset of $X$. We say that $E$ and $K$ are strictly associated if there exists a constant $\kappa>0$ such that

$$
\|f\|_{A} \leqq \kappa\left\|\xi_{K} f\right\|_{U} \text { for all } f \in \operatorname{Trig}_{E}(G)
$$

where $\xi_{K}$ denotes the characteristic function of $K$. In particular if $E$ and $G$ are strictly associated, we say that $E$ is a Sidon set. We say that $E$ and $K$ are associated if $E \backslash F$ and $K$ are strictly associated for some finite subset $F$ of $E$. 
Definition (see López and Ross [6] p. 110). Let $X_{0}$ denote a subgroup of an abelian group $X$. A subset $E$ of $X$ is called $X_{0}$ subtransversal provided that each coset of $X_{0}$ intersects $E$ in at most one point. We say that $E$ is almost $X_{0}$-subtransversal if $E \backslash F$ is $X_{0}$ subtransversal for some finite subset $F$ of $E$.

Theorem 1.3 (see [6] Theorem 8.22, López and Ross). A Sidon set $E$ is strictly associated with every compact subset of $G$ with nonvoid interior if and only if $E$ is $X_{0}$-subtransversal for all finite subgroups $X_{0}$ of $X$.

Ross proved the above theorem after previously showing that the theorem was true if the property "strictly associated" is replaced with "associated" and the property " $X_{0}$-subtransversal" is replaced with "almost $X_{0}$-subtransversal." Both results are generalizations of previous results due to Déchamps-Gondim [1, 2,3] which show the theorems to work for $G$ connected. In this case, the condition that $E$ be $X_{0}$-subtransversal for each finite subgroup $X_{0}$ of $X$ is trivial since $X$ is torsion free and has only one finite subgroup, $\{1\}$.

In this paper, a characterization is given of the Sidon sets which are associated with a given compact subset $K$ of $G$ for which $\lambda K=$ $\lambda($ int $K)>0$. In $\S 2$, we define the notion of a $K$-basic subset of $X$ and establish several elementary properties. In $\S 3$, we show that Sidon $K$-basic sets are those which are associated with $K$.

2. Definition and properties of $K$-basic sets.

DEFinition 2.1. Let $K$ be a nonvoid compact subset of $G$ and let $E$ be a subset of $X$. We call $E$ a $K$-basic set if the zero function is the only function, $f \in \operatorname{Trig}_{E}(G)$ such that $\xi_{K} f=0$. We denote by $D(K)$, the set of all $K$-basic sets and we let $D_{1}(K)=\{E \in D(K): 1 \in E\}$.

2.2. In the following lemma, we list without proof certain easy consequences of the definition.

Lemma. Let $K, K_{1}$ be compact subsets of $G$. Then the following are true.

(i) Subsets of K-basic sets are K-basic sets.

(ii) $A K$-basic set is a $K+a$-basic set for each $a \in G$.

(iii) If $K_{1} \supseteqq K$, then a $K$-basic set is a $K_{1}$-basic set.

(iv) Translates of $K$-basic sets are $K$-basic sets. In particular, for each $E \in D(K)$ and each $\chi \in E$, we have $\chi^{-1} E \in D_{1}(K)$.

(v) Inverses of $K$-basic sets are $K$-basic sets. 
2.3. We now characterize the property of a set $E \subseteq X$ being $X_{0}$-subtransversal for all finite subgroups $X_{0}$ of $X$ in terms of the ideas introduced in 2.1.

THEOREM. Let $E$ be a subset of $X$. Then the following are equivalent. of $X$.

(i) The set $E$ is $X_{0}$-subtransversal for all finite subgroups $X_{0}$

(ii) The set $E$ is a $K$-basic set for all compact subsets $K$ of $G$ having positive Haar measure.

(iii) For some compact set $K_{1}$ having positive Haar measure, the set $E$ is a $K$-basic set for all compact sets $K \subseteq K_{1}$ having positive Haar measure.

Proof. That (i) implies (ii) is a simple consequence of López and Ross 8.12. Now suppose that (i) does not hold. Then there exist characters $\chi_{1}$ and $\chi_{2}$ of finite order such that $\chi \chi_{1}$ and $\chi \chi_{2}$ are elements of $E$ for some $\chi \in X$. The set $K=\left\{x \in G: \chi_{1}(x)=\chi_{2}(x)=1\right\}$ is a closed neighbourhood of the origin in $G$ on which the $E$-spectral polynomial $\chi \chi_{1}-\chi \chi_{2}$ is identically zero. We conclude that $E$ is not a $K$-basic set. It is clear that (ii) implies (iii). Suppose that (iii) holds and let $K_{2}$ be any compact set having positive Haar measure. We note that the function $\xi_{-K_{2}} * \xi_{K_{1}}: a \rightarrow \lambda\left(\left(K_{2}+a\right) \cap K_{1}\right)$ is a nontrivial continuous function and hence $\lambda\left(\left(K_{2}+\alpha\right) \cap K_{1}\right)>0$ for some $a \in G$. Hence $E$ is a $\left(K_{2}+a\right) \cap K_{1}$-basic set by (iii). Noting 2.2(ii) and 2.2(iii) and the fact that $K_{2} \supseteqq\left(K_{2}+a \cap K_{1}\right)-a$, we conclude that $E$ is a $K_{2}$-basic set.

COROLLARY 2.4. If $G$ is a connected compact abelian group, then each subset $E$ of $X$ is a K-basic set for all compact subsets $K$ of $G$ having positive Haar measure.

Proof. As noted in 1.3, condition (i) of the previous theorem is trivially satisfied for all subsets $E$ of $X$.

2.5. We now show that each set $E \subseteq X$ possesses at least one maximal $K$-basic subset and all maximal $K$-basic sets $E$ possess a characteristic property.

THEOREM. Let $E$ be a subset of $X$ and $K$ a compact subset of G. Then the following is true.

(i) The set $E$ contains a maximal $K$-basic subset $E_{\text {n. }}$

(ii) $A$ subset $E_{0}$ of $E$ is a maximal $K$-basic set if and only if for each $f \in \operatorname{Trig}_{E}(G)$, there exists a unique polynomial $f_{0} \in \operatorname{Trig}_{E_{0}}(G)$ such that $\xi_{K}\left(f-f_{0}\right)$ is the zero function. 
Proof. To show (i), let $\mathscr{C}=\left\{E_{1} \in D(K): E_{1} \subseteq E\right\}$ and order $\mathscr{C}$ by inclusion. Note that $\varnothing \in D(K)$. It is easily seen that each ascending chain $\left\{E_{\alpha}\right\}$ of sets in $\mathscr{C}$ is bounded above by $\bigcup_{\alpha} E_{\alpha}$ and hence by Zorn's lemma, the set $\mathscr{C}$ has a maximal element.

Now let $E_{0}$ be a maximal $K$-basic subset of $E$. Assume firstly that some nonzero element $f \in \operatorname{Trig}_{E}(G)$ has the property that $\xi_{K}\left(f-f_{0}\right)$ is nonzero for each $f_{0} \in \operatorname{Trig}_{E_{0}}(G)$. If we set $f=\sum a_{\chi} \chi$ where each $\chi \in E$, then we deduce that for some $\chi \in E$, we have $\xi_{K}\left(\chi-f_{0}\right)$ is nonzero for all $f_{0} \in \operatorname{Trig}_{E_{0}}(G)$. It follows readily that $E_{0} \cup\{\chi\}$ is a $K$-basic set contradicting the maximality of $E_{0}$. Now assume that for some $f \in \operatorname{Trig}_{E}(G)$, there exist distinct polynomials $f_{1}$ and $f_{2}$ belonging to $\operatorname{Trig}_{E_{0}}(G)$ such that $\xi_{K}\left(f-f_{1}\right)=\xi_{K}\left(f-f_{2}\right)=0$. It follows that $\xi_{K}\left(f_{1}-f_{2}\right)=0$ contradicting the fact that $E_{0}$ is a $K$-basic set.

On the other hand, if $E_{0}$ is any subset of $E$ having the property that for each $f \in \operatorname{Trig}_{E}(G)$, there exists a unique element $f_{0} \in \operatorname{Trig}_{E}(G)$ such that $\xi_{K}\left(f-f_{0}\right)=0$, then $E_{0}$ is a $K$-basic set because a polynomial $f_{0} \in \operatorname{Trig}_{E_{0}}(G)$ such that $\xi_{K} f_{0}=0$ is necessarily the zero polynomial since $\xi_{K}\left(f_{0}-f\right)=0$ for $f=f_{0}$ and $f=0$. Further, $E_{0}$ is maximal since if $\chi \notin E_{0}$ then $\xi_{K}\left(\chi-f_{0}\right)=0$ for some $f_{0} \in \operatorname{Trig}_{E_{0}}(G)$ and hence $E_{0} \cup\{\chi\}$ is not a $K$-basic set.

\section{Characterization of sets associated with $K$.}

3.1. In this section, we show by a modification of DéchampsGondim's arguments that $E$ is a $K$-basic Sidon set if and only if $E$ and $K$ are associated where $K$ is a compact set such that $\lambda K=$ $\lambda($ int $K)>0$. We commence with the following theorem.

THEOREM. Let $K$ be a compact subset of $G$ with nonvoid interior and let $m \in N$. Then there exists a positive constant $\delta(m, K)$ such that for all $K$-basic sets $E$ having at most $m$ elements and for all $f \in \operatorname{Trig}_{E}(G)$, we have

$$
\int_{G}\left|\xi_{K} f\right|^{2} d \lambda \geqq \delta(m, K) \sum_{\chi \in E}|f(\chi)|^{2} .
$$

The proof imitates the classical proof by Zygmund [8, p. 60] which was observed to work for connected groups by DéchampsGondim [3, Lemme 6.3]. Connectedness is used specifically to show that for a certain nonzero polynomial $f$, we have $\int_{K_{1}} f d \lambda \neq 0$ for some $K_{1} \subseteq K$. For the above theorem, this will continue to hold since the polynomial $f$ will be an $E$-spectral polynomial for some $K$-basic set $E$. 
3.2. A critical argument which is needed later requires the existence of a unique solution to a system of linear equations. The following corollary to 3.1 which is a modified version of Lemme 6.4 of Déchamps-Gondim [3] provides this.

COROLLARY. Let $K$ be a compact subset of $G$ with nonvoid interior and let $m$ be a positive integer greater than one. Let $g$ be a nonnegative integrable function such that $\lambda(K \backslash \operatorname{supp} g)<m^{-2} \delta(m, K)$ where $\delta(m, K)$ is the constant appearing in 3.1 and let $\left\{\chi_{j}: j=\right.$ $1,2, \cdots, r\}$ be a K-basic set where $2 \leqq r \leqq m$. We set

$$
K_{n}=\left\{x \in K: g(x)>n^{-1}\right\}
$$

and let $H$ be the $r \times r$-matrix

$$
H_{j k}=\hat{g}\left(\chi_{j} \chi_{k}^{-1}\right) \text {. }
$$

For each $n$, such that $\lambda\left(K \backslash K_{n}\right)<m^{-2} \delta(m, K)$, we have

$$
\operatorname{det} H \geqq \delta_{n} \text { where } \delta_{n}=\min \left\{1, n^{-m}\left(\delta(m, K)-m^{2} \lambda\left(K \backslash K_{n}\right)\right)^{m}\right\} \text {. }
$$

Proof. Since $H$ is an hermitian matrix, there exists a diagonal matrix $D=\left\{\delta_{j_{k}} d_{j}\right\}_{j k}^{r}$ and a unitary matrix $U=\left\{u_{j_{k}}\right\}_{j k}^{r}$ such that $D=$ $U H U^{*}$. We calculate:

$$
\begin{aligned}
\operatorname{det} H & =\prod_{j=1}^{r} d_{j} \\
& =\prod_{j=1}^{r} \sum_{k, s}^{r} u_{j k} H_{k s} \overline{u_{j_{s}}} \\
& =\prod_{j=1}^{r} \sum_{k, s}^{r} u_{j_{k}} \overline{u_{j s}} \hat{g}\left(\chi_{k} \chi_{s}^{-1}\right) \\
& =\prod_{j=1}^{r} \int_{G} g\left(\sum_{k, s}^{r} u_{j_{k}} \overline{u_{j s}} \chi_{s} \chi_{k}^{-1}\right) d \lambda \\
& =\prod_{j=1}^{r} \int_{G} g\left|\sum_{s}^{r} \overline{u_{j s} \chi_{s}}\right|^{2} d \lambda \\
& \geqq \prod_{j=1}^{r} \int_{G} \xi_{K} g\left|\sum_{s}^{r} \overline{u_{j s}} \chi_{s}\right|^{2} d \lambda .
\end{aligned}
$$

We have the estimate for $j=1,2, \cdots, r$ :

$$
\begin{aligned}
\int_{G} \xi_{K} g & \left|\sum_{s} \overline{u_{j_{s}}} \chi_{s}\right|^{2} d \lambda \\
& \geqq \int_{G} \xi_{K_{n}} g\left|\sum_{s} \overline{u_{j_{s}}} \chi_{s}\right|^{2} d \lambda \\
& \geqq n^{-1} \int_{G} \xi_{K}\left|\sum_{s} \overline{u_{j_{s}}} \chi_{s}\right|^{2} d \lambda
\end{aligned}
$$




$$
\begin{aligned}
& \quad-n^{-1} \int_{G} \xi_{K^{\prime} K_{n}}\left|\sum_{s} \overline{u_{j s}} \chi_{s}\right|^{2} d \lambda \\
& \geqq n^{-1} \delta(m, K)-m^{2} n^{-1} \lambda\left(K \backslash K_{n}\right) \\
& \text { by the previous theorem } \\
& =n^{-1}\left(\delta(m, K)-m^{2} \lambda\left(K \backslash K_{n}\right)\right) .
\end{aligned}
$$

The corollary follows.

It is clear that a modification of Déchamps-Gondim's arguments is needed in the above theorem since the set $\left\{\chi_{j}: j=1,2, \cdots, r\right\}$ is not necessarily a $K_{n}$-basic set and hence we do not necessarily have the inequality

$$
\int_{G} \xi_{K_{n}}\left|\sum_{s} \overline{u_{j_{s}}} \chi_{s}\right|^{2} d \lambda \geqq \delta(m, K)
$$

3.3. We need to be able to choose a suitable nonnegative integrable function which, in particular, satisfies the requirement of 3.2. Hence we have

Lemma. Let $K$ be a compact subset of $G$ such that $\lambda K=\lambda$ int $K>0$ and let $\varepsilon>0$. There exists a nonnegative function $g \in A(G)$ such that supp $g \subseteq K$ and $\lambda\{x \in K: g(x) \neq 1\}<\varepsilon$.

Proof. Let $V=\operatorname{int} K$ and choose a compact set $F \leqq V$ such that $\lambda(V \backslash F)<\varepsilon$. Since $A(G)$ is a normal family of functions on $G$ (see Hewitt and Ross [5](34.21)), it contains a function $g$ such that $g(x)=1$ for $x \in F$ and $g(x)=0$ for $x \in G \backslash V$.

Definition 3.4 (see López and Ross [6] 7.3). Let $E$ be a nonvoid symmetric subset of $X$ and $K$ a nonvoid compact subset of $G$. Then we say that $E$ is an $F Z(K)$-set if there exists a positive constant $\kappa$ and a finite symmetric subset $F$ of $E$ such that for all $f \in \operatorname{Trig}_{E \mid F}^{r}(G)$ we have

$$
\|f\|_{A} \leqq \kappa\left\|\xi_{K} f^{+}\right\|_{u}
$$

3.5 We have the following analogue of 8.16 of López and Ross [6].

THEOREM. Let $K$ be a compact subset of $G$ such that $\lambda(K)=$ $\lambda($ int $K)>0$. A symmetric subset $E$ of $X$ is an $F Z(K)$-set if and only if

and

(i) $E$ is a Sidon set

(ii) $E$ is the union of a symmetric finite set and a K-basic set. 
Proof. Suppose that $E$ is a $F Z(K)$-set. As observed in the proof of 8.3 of López and Ross [6], the statement (1) implies that $E \backslash F$ and $K$ are strictly associated and hence, in particular, the set $E \backslash F$ is a Sidon set. Since the set $F$ is finite, we conclude that $E$ is a Sidon set. Further the set $E \backslash F$ is a $K$-basic set since the existence of a nonzero $E \backslash F$-spectral trignometric polynomial $f$ such that $\xi_{K} f$ is the zero function would violate the condition that $E \backslash F$ and $K$ are strictly associated.

To prove converse, it is sufficient to show that each symmetric $K$-basic Sidon set $E$ is an $F Z(K)$-set. Noting 3.3, we choose a nonnegative function $g \in A(G)$ such that supp $g \subseteq-K$ and

$$
\lambda\{x \in-K: g(x) \neq 1\}<m^{-2} \delta(m, K)
$$

where $m$ is the positive integer appearing in 8.9 of López and Ross [6]. A slightly modified version of the argument used in 8.16 of López and Ross [6] can now be used to show that $E$ is an $F Z(K)$ set. In place of (8.15) of López and Ross [6] we have (3.2) and we let $\delta=\delta_{2}$. The set $F_{0}$ which López and Ross choose at the top of page 129 can be taken to be the empty set in our proof. The result follows.

3.6. Similar to 8.18 of López and Ross [6], we have the following.

Corollary. Let $K$ be as in 3.5 and let $E \subseteq X$. The following are equivalent.

(i) The set $E$ is associated with $K$.

(ii) The set $E$ is the union of a $K$-basic Sidon set and $a$ finite set.

Proof. The argument used in 3.5 suffices to show that (i) implies (ii). In order to show the reverse implication, we use the device used in 8.18 of López and Ross [6] which was first used by Drury. Let $G_{0}=G \times T$ and let $Y=X \times Z$ be its character group. Let $E$ be a $K$-basic Sidon subset of $X$ and let $E_{0}=E \times\{1\}$. We show that $E_{0} \cup E_{0}^{-1}$ is a $K \times T$-basic Sidon set. That it is a Sidon set is observed in 8.18 of López and Ross [6]. Suppose that $f=\sum_{j} \alpha_{j}\left(\chi_{j}, 1\right)+$ $\sum_{j} b_{j}\left(\dot{\phi}_{j}^{-1},-1\right)$ is an $E_{0} \cup E_{0}^{-1}$-spectral trignometric polynomial such that $\xi_{K \times T} f=0$. We have $z \xi_{K} \sum a_{j} \chi_{j}+\bar{z} \xi_{K} \sum b_{j} \phi_{j}^{-1}=0$ for all $z \in T$. This implies that $\xi_{K} \sum_{j} a_{j} \chi_{j}=\xi_{K} \sum_{j} b_{j} \phi_{j}^{-1}=0$. Since $E$ and $E^{-1}$ are $K$-basic sets (note 2.2(v)), we conclude that $\alpha_{j}=b_{k}=0$ for each $j, k$. Thus we see that $E_{0} \cup E_{0}^{-1}$ is a $K \times T$-basic set. The statement (i) follows from 3.5 using the argument appearing in 8.18 of López and Ross [6]. 
REMARKS 3.7. 1. We are unable to show that condition (i) in 3.6 can be replaced with the statement that $E$ and $K$ are strictly associated. In particular, 8.21 of López and Ross [6] admits no easy generalization.

2. In view of 8.24 of López and Ross [6], the assertion that, if $K$ is a compact subset of $G$ with int $K \neq \varnothing$, then a $K$-basic Sidon set is associated with $K$, is probably false.

\section{REFERENCES}

1. M. Déchamps-Gondim, Compacts associés à un ensemble de Sidon, C. R. Acad. Sci. Paris, 271A (1970), 590-592.

2. - Sur les ensembles de Sidon topologiques, C. R. Acad. Sci. Paris, 271A (1970), 1247-1249.

3. - Ensembles de Sidon topologiques, Ann. Inst. Fourier (Grenoble) 22, Fasc., 3 (1972), 51-79.

4. S. W. Drury, The Fatou-Zygmund property for Sidon sets, Bull. Amer. Math. Soc., 80 (1974), 535-538.

5. E. Hewitt and K. A. Ross, Abstract Harmonic Analysis, Vol. I, II, Springer-Verlag, Heidelberg, 1963, 1970.

6. J. A. López and K. A. Ross, Sidon sets, Lecture notes in pure and applied mathematics, Vol. 13, Marcel Dekker, 1975.

7. K. A. Ross, Sur les compacts associés à un ensemble de Sidon, C. R. Acad. Sci. Paris, 275A (1972), 185-185.

8. A. Zygmund, On a theorem of Hadamard, Ann. Soc. Polon. Math., 21 (1948), 52-69 Errata, 357-358.

Received November 2, 1976.

UNIVERSITY OF MELBOURNE

PARKVILLE, ViCTORIA 3052

AUSTRALIA 


\section{PACIFIC JOURNAL OF MATHEMATICS}

\section{EDITORS}

RICHARD ARENS (Managing Editor)

University of California

Los Angeles, California 90024

C. W. CURTIS

University of Oregon

Eugene, OR 97403

C. C. MOORE

University of California

Berkeley, CA 94720

\section{J. DugundJI}

Department of Mathematics University of Southern Californıa Los Angeles, California 90007

R. FinN AND J. Milgram Stanford University Stanford, California 94305

\section{ASSOCIATE EDITORS}

E. F. BECKENBACH

B. H. NEUMANN

F. WOLF

K. YOSHIDA

\section{SUPPORTING INSTITUTIONS}

UNIVERSITY OF BRITISH COLUMBIA CALIFORNIA INSTITUTE OF TECHNOLOGY UNIVERSITY OF CALIFORNIA MONTANA STATE UNIVERSITY UNIVERSITY OF NEVADA, RENO NEW MEXICO STATE UNIVERSITY OREGON STATE UNIVERSITY UNIVERSITY OF OREGON OSAKA UNIVERSITY
UNIVERSITY OF SOUTHERN CALIFORNIA STANFORD UNIVERSITY UNIVERSITY OF TOKYO UNIVERSITY OF UTAH WASHINGTON STATE UNIVERSITY UNIVERSITY OF WASHINGTON AMERICAN MATHEMATICAL SOCIETY NAVAL WEAPONS CENTER 


\section{Pacific Journal of Mathematics}

\section{Vol. 71, No. $1 \quad$ November, 1977}

Charalambos D. Aliprantis and Owen Sidney Burkinshaw, On universally complete Riesz spaces ............................. 1

Stephen Richard Bernfeld and Jagdish Chandra, Minimal and maximal solutions of nonlinear boundary value problems .................

John H. E. Cohn, The length of the period of the simple continued fraction of

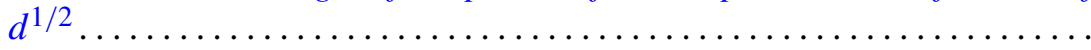

Earl Vern Dudley, Sidon sets associated with a closed subset of a compact abelian group .................................... 33

Larry Finkelstein, Finite groups with a standard component of type $J_{4} \ldots \ldots$

Louise Hay, Alfred Berry Manaster and Joseph Goeffrey Rosenstein, Concerning partial recursive similarity transformations of linearly ordered sets .......................................

Richard Michael Kane, On loop spaces without $p$ torsion. II ............

William A. Kirk and Rainald Schoneberg, Some results on

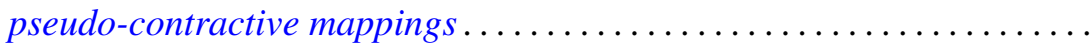

Philip A. Leonard and Kenneth S. Williams, The quadratic and quartic character of certain quadratic units. I. .

Lawrence Carlton Moore, A comparison of the relative uniform topology and the norm topology in a normed Riesz space .................

Mario Petrich, Maximal submonoids of the translational hull 119

Mark Bernard Ramras, Constructing new R-sequences . . .

Dave Riffelmacher, Multiplication alteration and related rigidity properties

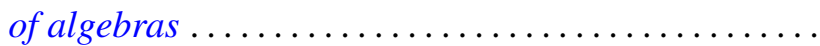

Jan Rosiński and Wojbor Woyczynski, Weakly orthogonally additive functionals, white noise integrals and linear Gaussian stochastic processes.

Ryōtarō Satō, Invariant measures for ergodic semigroups of operators

Peter John Slater and William Yslas Vélez, Permutations of the positive integers with restrictions on the sequence of differences...

Edith Twining Stevenson, Integral representations of algebraic cohomology classes on hypersurfaces ........................

Laif Swanson, Generators of factors of Bernoulli shifts . 